\title{
Dose Perturbations of Gold Fiducial Markers in the Prostate Cancer Intensity Modulated Proton Radiation Therapy (IMPT)
}

\author{
Miao Zhang ${ }^{1}$, Sung Kim ${ }^{1}$, Ting Chen ${ }^{1}$, Xiaohu Mo ${ }^{2}$, Bruce G. Haffy ${ }^{1}$, Ning J. Yue ${ }^{1}$ \\ ${ }^{1}$ Department of Radiation Oncology, The Cancer Institute of New Jersey, \\ UMDNJ-Robert Wood Johnson University Hospital, New Brunswick, USA \\ ${ }^{2}$ Medical Physics Department, University of Wisconsin-Madison, Madison, USA \\ Email: zhangm3@umdnj.edu
}

Received April 7, 2012; revised May 13, 2012; accepted May 22, 2012

\begin{abstract}
The objective of this study is to investigate the dose perturbations introduced by the implanted gold fiducial markers in the prostate cancer intensity modulated proton therapy (IMPT) and the impacts of different plan designs on the perturbations. Five proton plans: a single lateral field 3D-modulation (3D-mod) plan, 2 fields laterally opposing 3D-mod plan, 6-, 9-, and 18-field distal edge tracking (DET) plans were designed on the CT images of a prostate patient. The dose distributions were first generated for the plans free of fiducial markers with 78 Gy prescribed to $95 \%$ of the PTV. To derive the dose perturbations of the gold fiducial markers, three cylindrical shaped gold fiducial markers $(3 \mathrm{~mm}$ long and $1 \mathrm{~mm}$ in diameter) were artificially inserted into the prostate, and the dose distributions were re-computed. Monte Carlo method was used for dose computation. It was found that the gold fiducial markers perturbed the dose distributions, especially along the beam paths. The markers caused a shadowing effect reducing the doses in the areas beyond the markers. Overall, due to the presence of the fiducial markers, $\mathrm{D}_{99 \%}$ of prostate were reduced by $2.96 \mathrm{~Gy}, 4.21 \mathrm{~Gy}$, $0.16 \mathrm{~Gy}, 0.34 \mathrm{~Gy}, 0.15 \mathrm{~Gy}$ for the plans of single field 3D-mod, 2-field parallel opposed 3D-mod, 6-, 9-, and 18-field DET respectively. Our study showed these dose perturbation effects decreased with the increase of number of beam angles. Up to 6 beam angles may be required to reduce the dose perturbations from the gold fiducial markers to a clinically acceptable level in IMPT.
\end{abstract}

Keywords: Gold Fiducial Marker; Intensity Modulated Proton Therapy; Prostate Cancer; Monte Carlo

\section{Introduction}

In prostate radiotherapy, high density fiducial markers are often used on a daily basis for accurate localization of prostate gland. While high density fiducial markers are preferred for providing high contrast images, the presence of these high density markers may perturb the radiation field and introduce undesired dose distributions. For conventional megavoltage photon radiotherapy, both theoretical and experimental studies have shown the presence of small high density materials, like gold, does not introduce significant dose perturbations due to the strong penetrating power of megavoltage photons and the multiple beam angles arrangement [1]. However, the use of high density fiducial markers in proton therapy may require careful consideration [2-5]. Newhauser et al. [2] used the Monte Carlo method to calculate the dosimetric effects caused by cylindrical shaped gold fiducial markers ( $3 \mathrm{~mm}$ long and $0.9 \mathrm{~mm}$ in diameter) in a single direction spread out Bragg peak (SOBP) proton field. Com- pared to the dose distribution without markers in water, they found that gold markers casted a "dose shadow" to the area behind the markers. The magnitude of the underdose depended on the orientation of the gold markers with respect to the radiation field, as well as the relative locations of the gold markers to the distal edge of the SOBP. In the worst case scenario (marker was parallel to the beam direction and close to the distal end of the SOBP) the under-dose to the tissue behind the marker could be as large as $50 \%$. Even when the orientation of the marker was perpendicular to the beam direction, the under-dose to the tissue behind the marker was still around $20 \%$. In a later investigation, Lim et al. [6] confirmed those Monte Carlo results by using diode detectors to measure the dose distributions in a water phantom.

Recently, more sophisticated proton delivery systems become available. The introduction of the active scanning technology in the proton delivery system allows 
people to conduct intensity modulated proton therapy (IMPT) by delivering intensity modulated proton pencil beams. With this relatively new delivery technique and potentially plans of more beam angles, the dose variation introduced by the gold fiducial markers may behave differently in the IMPT than in the passive scattered proton therapy. In this study, we used Monte Carlo dose calculation to investigate the dosimetric impact of gold fiducial markers in different prostate IMPT plans. Prostate dose volume histograms (DVHs) and equivalent uniform doses (EUDs) were calculated to evaluate the clinical dosimetry impacts to the target volume by the presence of the gold fiducial markers.

\section{Materials and Methods}

The simulation CT images of a prostate patient were used for the planning and dose calculation purposes. The prostate gland was delineated from the CT images as the clinical target volume (CTV). The planning target volume (PTV) was generated by adding $8 \mathrm{~mm}$ uniform margin around the CTV (Figure 1). Three cylindrical shaped gold fiducial markers ( $3 \mathrm{~mm}$ long and $1 \mathrm{~mm}$ in diameter) were artificially placed into the prostate on the CT images with the markers oriented along the superior-inferior direction. This type of gold fiducial marker has been routinely used in clinical radiation treatments of prostate cancers [7]. Five types of IMPT plans were generated: single lateral field plan with 3D-modulation (3D-mod) [8], 2-field parallel-opposed 3D-mod plan, and three Distal Edge Tracking (DET) [9] plans that respectively consisted of 6,9 , and 18 evenly spaced beams. The five plans were first generated free of markers with the prescription dose of 78 Gy to cover $95 \%$ of the PTV.

The dose distributions were calculated with an inhouse-developed proton planning system based on Geant 4 Monte Carlo code [10]. In the two 3D-mod plans, a 3dimentional spots grid with uniform $3 \mathrm{~mm}$ spot grid resolution were planned to cover the entire target. For the three DET plans, only spots falling on the distal edge of the PTV were irradiated from a given beam angle. The pencil beams in both cases were mono-directional with a lateral intensity profile following a Gaussian distribution.

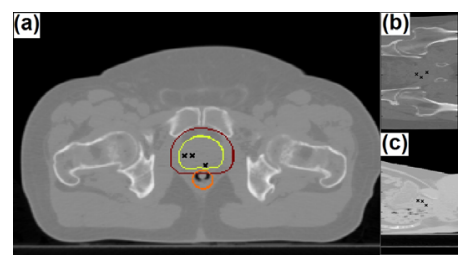

Figure 1. Transverse (a), Coronal (b), and Sagittal (c) view of a slice of the patient's CT image. The prostate, PTV, and rectum are represented by the yellow, red, and orange contours. The cross marks show the location of artificially embedded gold fiducial markers projected on the slice.
The standard deviation $\sigma$ of the Gaussian distribution was $3 \mathrm{~mm}$ in air at the iso-center. In the calculations, tissue heterogeneity was taken into account and corrected by converting the CT pixels into different materials of different density following the values in Table 1. For each pencil beam, $1 \times 10^{5}$ proton histories were simulated. At least $1 \times 10^{8}$ proton histories were used for each of the 5 simulated plans. The dose calculation grid was $1 \mathrm{~mm} \times 1$ $\mathrm{mm} \times 3 \mathrm{~mm}$.

The plans were recomputed after the three gold fiducial markers were artificially embedded into the CT images under identical beam conditions to the corresponding original plans. The perturbations introduced by the gold fiducial markers were derived by comparing the differences without and with the presence of the markers. Both the prostate DVHs and equivalent uniform dose (EUD) were used to compare the differences. The EUD was calculated to evaluate the radiobiological significance. The EUD was computed with equation [11]:

$$
\begin{gathered}
\mathrm{EUD}=\frac{N}{d_{r e f}}\left[-\frac{\alpha}{\beta}+\sqrt{\left.\left(\frac{\alpha}{\beta}\right)^{2}+4 \cdot \frac{d_{r e f}}{N}\left(\frac{\alpha}{\beta}+d_{r e f}\right) \frac{\ln A}{\ln S F_{2}}\right]}\right. \\
A=\sum_{i} v_{i} S F_{2}^{\frac{D_{i}}{d_{r e f}}}
\end{gathered}
$$

where $\mathrm{SF}_{2}$ is the cell survival fraction after a dose of 2 Gy. Following parameters were chosen for prostate EUD calculation: $\alpha / \beta=1.5, d_{\text {ref }}=2 \mathrm{~Gy}, S F_{2}=0.45$ [12-14].

\section{Results}

Before Dose distributions for the 3D-mod and DET plans, with and without the presence of the gold markers, are respectively shown in Figure 2. The gold fiducial markers introduced visible target under-dose in all the plans. The under-dose area followed a "streak" pattern which was along the direction of the beam angles marked by small arrows in the subplots. The lateral dose profiles displayed in the figure were used to demonstrate the significance of the marker induced under-dose in each of the

Table 1. Density threshold for Monte Carlo material construction. The material composition follows the NIST website definition [15].

\begin{tabular}{ll}
\hline \multicolumn{1}{c}{ Material } & Density range $\left(\mathrm{g} / \mathrm{cm}^{3}\right)$ \\
\hline Air & $<0.207$ \\
Adipose tissue & $0.207<\rho<0.979$ \\
Water & $0.979<\rho<1.0$ \\
Muscle & $1.0<\rho<1.109$ \\
Dense bone & $1.109<\rho<10$ \\
Gold & 19.3 \\
\hline
\end{tabular}




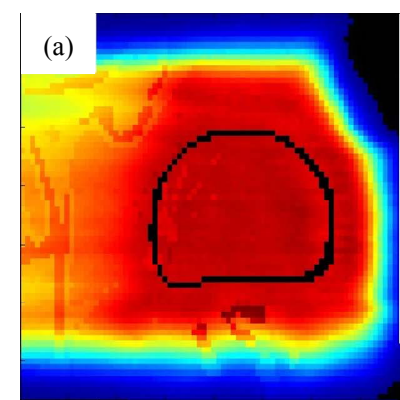

(b)

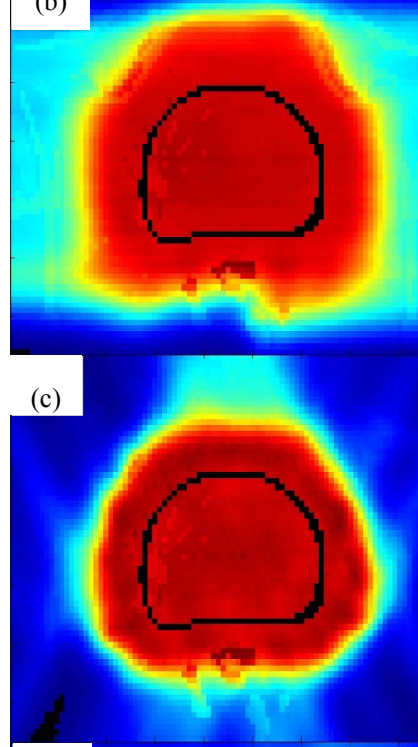

(d)

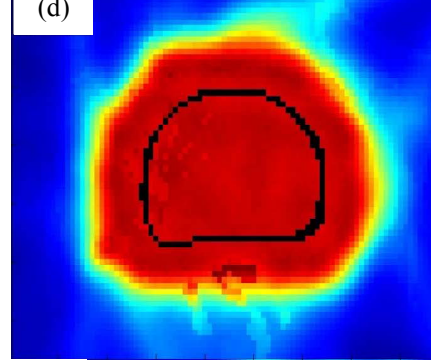

(e)

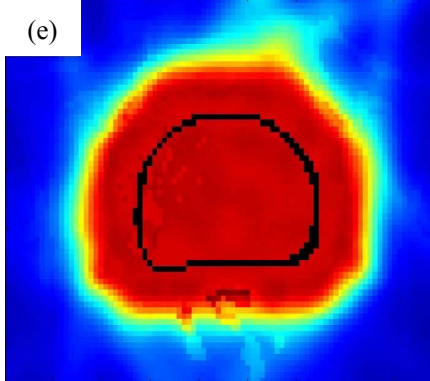

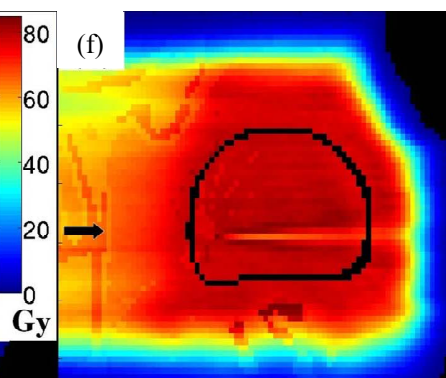

(g)
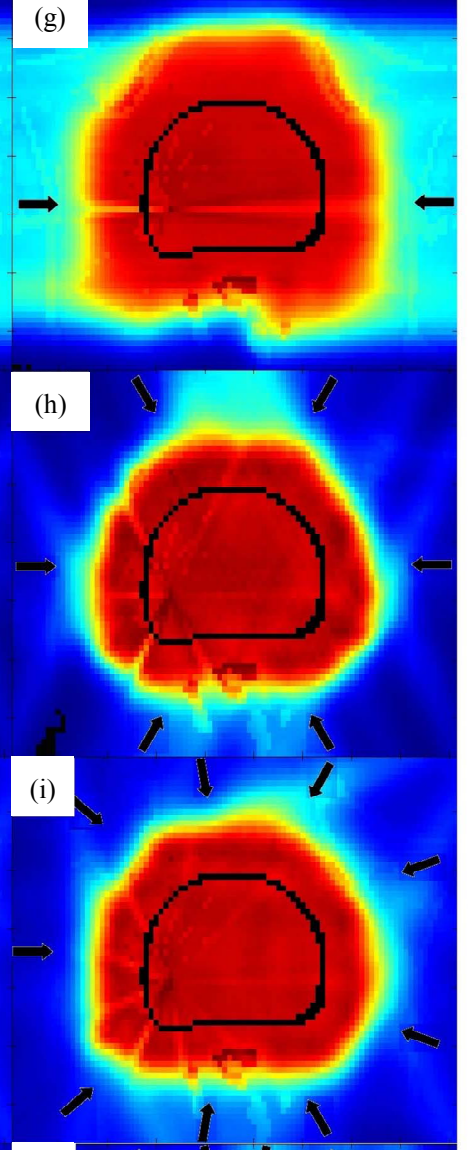

(j)
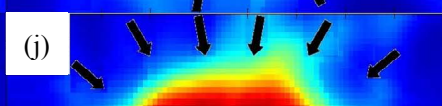

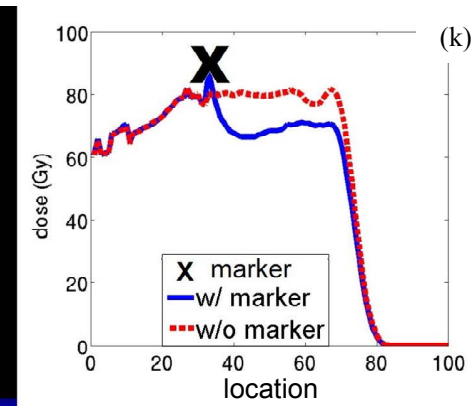

(k)
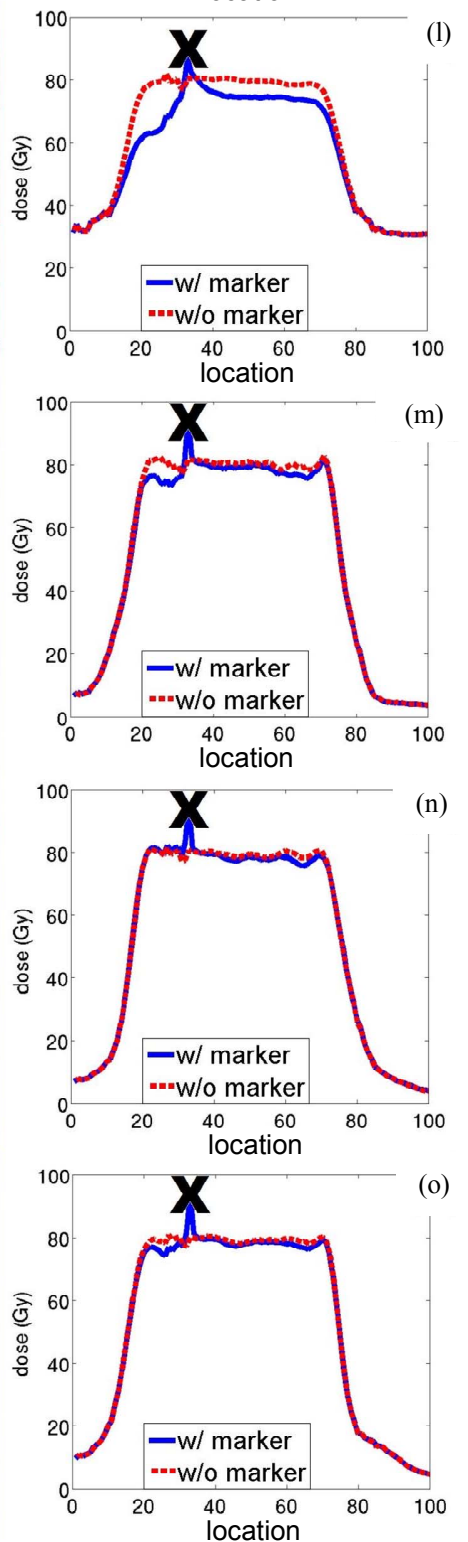

Figure 2. Planar dose distribution without (a)-(e) and with fiducial markers (f)-(j), and the lateral profile across one gold fiducial marker with and without fiducial markers (k-o). The subplots are arranged that five rows correspond to, from top to bottom, single lateral field 3D-mod, 2-field laterally opposing 3D-mod, 6-field DET, 9-field DET, and 18-field DET. Three columns correspond to, from left to right, the dose distribution without marker, the dose distribution with marker, and the lateral dose profiles across a gold marker. The color legend in (a) applies to all the planar dose distributions. The black arrows in the middle column show the gantry angle for each plan. The " $X$ " marks the locations of fiducial markers which correspond to the location of spikes in the lateral dose profile. The spikes are not hot spots in prostate but high dose to the fiducial marker themselves. 
plans. The spikes in the profiles were the locations of gold fiducial marker, which had higher dose due to its high density. The single field 3D-mod plan had around $20 \%$ under-dose to the prostate tissue behind the fiducial markers, whereas the 2-field laterally opposed 3D-mod plan produced around $20 \%$ under-dose to the prostate on the side closer to the edge of the target and about $10 \%$ under-dose in the side further away from the edge. In the 6-field DET plan, there was about $10 \%$ under-dose to the prostate tissue on the side closer to the edge of the target. In the 9-field and the 18-field DET plans, the maximum under-dose was less than $3 \%$ of the prescription dose.

The zoomed views on the low dose region of the prostate DVHs of each plan are shown in Figure 3. Prostate DVHs were nearly identical at the highest dose region regardless of the presence of the markers but started to deviate at lower doses. This means the gold fiducial markers did not create hot spots but introduced cold spots in the target. It is observed that the gold fiducial markers caused severe under-dose to a small fraction of the prostate volume in both the single field and the 2-field laterally opposed 3D-mod plan, but not in the DET plans. Table 2 summarizes the values of the prostate $D_{\min }, D_{99 \%}$, and EUDs for the plans with and without the presence of the markers and their differences. Compared to single and 2-field 3D-mod plans, multiple fields DET plans suffered much less dose perturbations due to the presence of the gold fiducial markers.
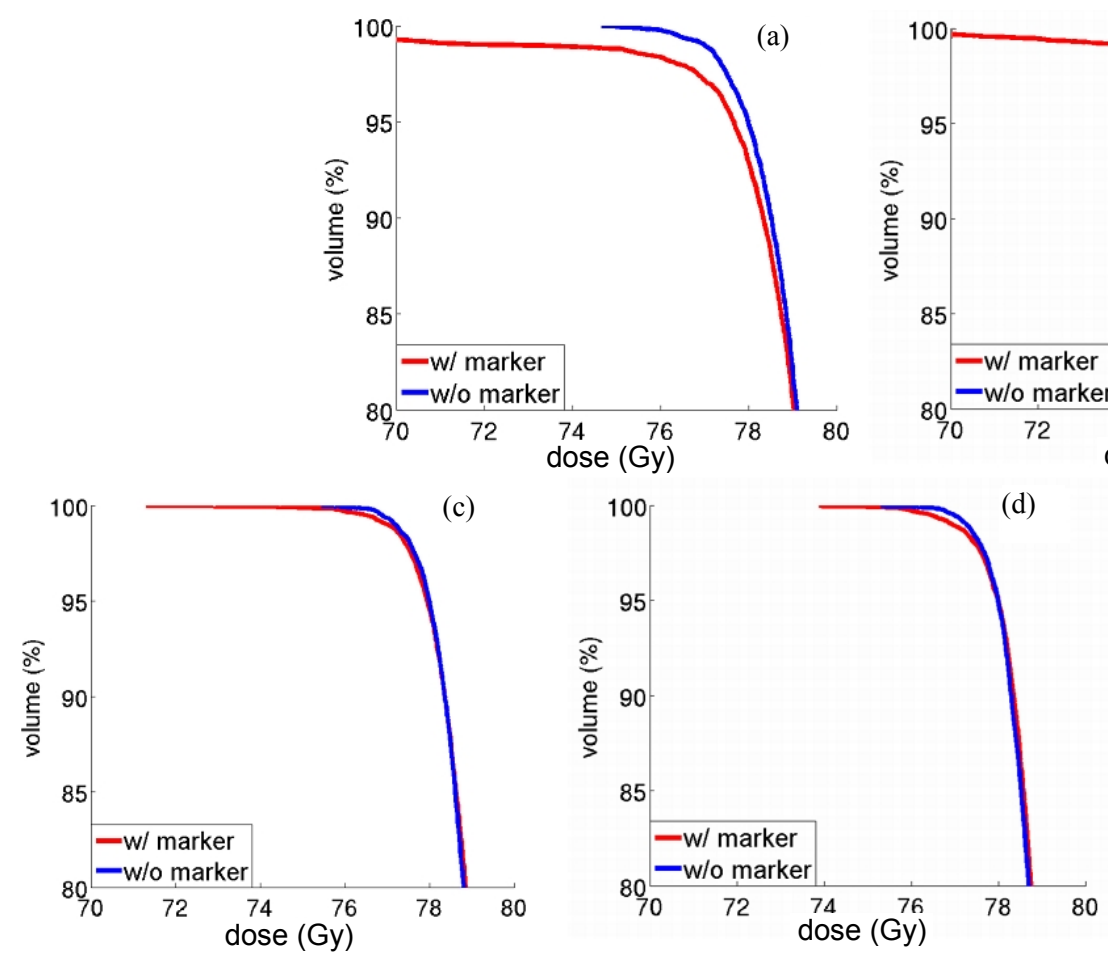

\section{Discussion}

Our results demonstrated that in prostate cancer IMPT, the use of gold fiducial markers would still introduce cold spots in the target similar to the passive scattered proton treatments [2-5]. The observed $10 \%$ to $20 \%$ under-dose to the tissue immediately next to the marker in the lateral dose profiles across the fiducial markers for the single field and the 2-field laterally opposed 3D-mod plans was in good agreement with previous published single SOBP field results $[2,6]$. It appears that the dose perturbations introduced by the use of gold fiducial mar-

Table 2. The prostate $D_{\text {min }}, D_{99 \%}$, and EUD from the planned and delivered dose distributions. The unit is in Gy.

\begin{tabular}{|c|c|c|c|c|c|c|}
\hline & & $\begin{array}{l}\text { single-field } \\
\text { 3D-mod }\end{array}$ & $\begin{array}{l}\text { 2-field } \\
\text { 3D-mod }\end{array}$ & $\begin{array}{l}\text { 6-field } \\
\text { DET }\end{array}$ & $\begin{array}{l}\text { 9-field } \\
\text { DET }\end{array}$ & $\begin{array}{c}\text { 18-field } \\
\text { DET }\end{array}$ \\
\hline \multirow{3}{*}{$D_{\min }$} & w/o marker & 74.66 & 75.45 & 75.45 & 75.31 & 75.05 \\
\hline & w/ marker & 62.22 & 67.62 & 71.31 & 73.89 & 74.30 \\
\hline & diff. & -12.44 & -8.83 & -4.14 & -1.42 & -0.75 \\
\hline \multirow{3}{*}{$\mathrm{D}_{99 \%}$} & w/o marker & 77.29 & 77.01 & 77.19 & 77.28 & 77.33 \\
\hline & w/ marker & 74.33 & 72.80 & 77.04 & 76.94 & 77.18 \\
\hline & diff. & -2.96 & -4.21 & -0.16 & -0.34 & -0.15 \\
\hline \multirow{3}{*}{ EUD } & w/o marker & 79.21 & 79.60 & 79.40 & 79.18 & 79.09 \\
\hline & $\mathrm{w} /$ marker & 77.49 & 73.31 & 79.38 & 79.21 & 79.16 \\
\hline & diff. & -1.71 & -6.29 & -0.02 & 0.03 & 0.07 \\
\hline
\end{tabular}
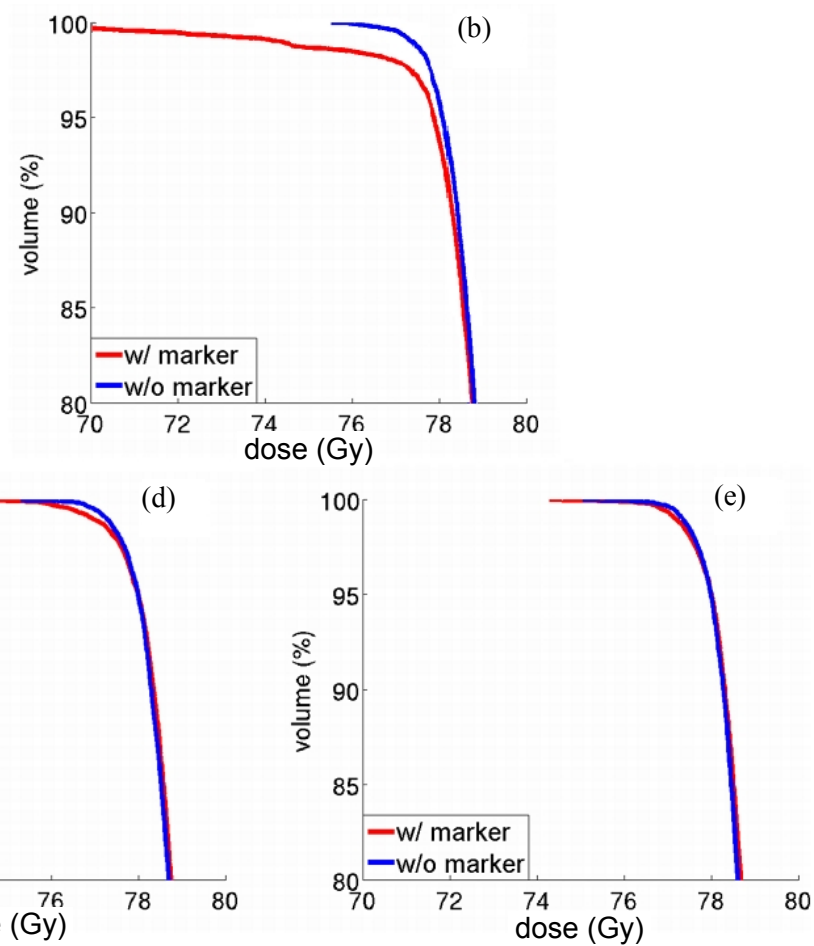

Figure 3. The focused view of the prostate DVHs at the low dose region from the single field 3D-mod (a); the 2-field laterally opposing 3D-mod (b); 6-field DET (c); 9-field DET (d); and 18-field DET (e) plans. 
kers do not depend on the delivery technique, whether it was passive scattering or active scanning, but the number of beams of different gantry angles. With the increase of beams, the dose perturbation decreased. If the number of beams increased to more than 6 , dose perturbation impacts on $D_{\min }$ and $D_{99 \%}$ would be less than $5 \%$ and $0.5 \%$, respectively, and may be clinically acceptable.

The prostate EUD calculation was an attempt to translate the prostate under-dose into a clinical subjective parameter by evaluating both the magnitude of the underdose and the affected volume. However, EUD should not be used as a solo parameter to make clinical decisions. Calculation of EUD depends on the choice of parameters, e.g., $\alpha / \beta$ and $S F_{2}$, and different choices of the parameters may result in different EUD values even for an identical dose distribution. Further, the EUD parameters available in the literature were derived by retrospectively looking at the relation between the dose distributions and the treatment outcomes [14]. Using selected parameters and equations to calculate EUD is essentially interpolating the pool of treated dose distributions used to derive the parameters to predict the possible treatment outcome from the current dose distribution. The EUD value can be clinically meaningful if the dose distribution to be calculated is similar to the ones used to derive the EUD parameters. However, for certain situations, such as the one presented in this paper where cold spots appeared in the center of the prostate, it is less likely that similar dose distributions were included in the pool of data used to derive the EUD parameters. Hence, the EUD value calculated here may not accurately predict the actual biological consequences. As an example, in the single field 3D-mod plan, the EUD of the delivered dose distribution was 77.49 Gy. The biological consequence of having a cold spot in the center of the prostate with a EUD of 77.49 Gy will not be the same as for another plan with EUD of 77.49 Gy but where the prostate was uniformly under-dosed by a few percent. Therefore, the EUD value presented in this study only served as a reference. With the current choice of parameters, using gold fiducial markers in the multiple field DET plans would not be a compromise.

With more beams of different gantry angles, proton treatments with DET technology will be more efficient than the 3D-mod in terms of delivery, since much fewer pencil beams are required for an individual field in DET than that in 3D-mod. On the other hand, the clinical feasibility of the proposed multiple field IMPT plan may be limited by the current proton machine design, such as slow gantry rotation and slow beam energy switch. However, with the technical advancement and machine design improvement, those limitations can be resolved.

Gold markers are the most frequently used fiducial markers in current practice. However, different types of markers have been introduced into clinical use, e.g., carbon coated ceramic (Carbon Medical Technologies, St. Paul, MN), stainless steel, titanium, or gold with coiled design (Visicoil; RadioMed Corp., Tyngsboro, MA) instead of a solid cylinder shape. Since the cylindrical gold markers investigated in this study have the highest density and atomic number compared to others, the simulated target under-dose may represent the worst possible situation.

\section{Conclusion}

Gold fiducial markers would introduce target under-dose in the prostate IMPT. With the increase of beam angles, the target under-dose decreased. It appears that 6 beams of evenly distributed gantry angles are adequate to reduce the dose perturbation to a clinically acceptable level.

\section{Acknowledgements}

Authors would like to thank Ms. Lydia Handsfield from University of Virginia and Dr. Songbing Qin from The first affiliated hospital of Soochow University, Suzhou, China for their insightful discussions on this project.

\section{REFERENCES}

[1] J. C. L. Chow and G. N. Grigorov, "Monte Carlo Simulations of Dose near a Nonradioactive Gold Seed," Medical Physics, Vol. 33, No. 12, 2006, pp. 4614-4621. doi:10.1118/1.2388573

[2] W. Newhauser, J. Fontenot, N. Koch, L. Dong, A. Lee, Y. Zheng, L Waters and R. Mohan, "Monte Carlo Simulations of the Dosimetric Impact of Radiopaque Fiducial Markers for Proton Radiotherapy of the Prostate," Physics in Medicine and Biology, Vol. 52, No. 11, 2007, pp. 2937-2952. doi:10.1088/0031-9155/52/11/001

[3] A. Giebeler, J. Fontenot, P. Balter, G. Ciangaru, R. Zhu and W. Newhauser, "Dose Perturbations from Implanted Helical Gold Markers in Proton Therapy of Prostate Cancer," Journal of Applied Clinical Medical Physics, Vol. 10, 2009, pp. 63-70.

[4] J. Cheung, R. J. Kudchadker, X. R. Zhu, A. K. Lee and W. D. Newhauser, "Dose Perturbations and Image Artifacts Caused by Carbon-Coated Ceramic and Stainless Steel Fiducials Used in Proton Therapy for Prostate Cancer," Physics in Medicine and Biology, Vol. 55, No. 23, 2010, pp. 7135-7147. doi:10.1088/0031-9155/55/23/S13

[5] J. Y. Huang, W. D. Newhauser, X. R. Zhu, A. K. Lee and R. J. Kudchadker, "Investigation of Dose Perturbations and the Radiographic Visibility of Potential Fiducials for Proton Radiation Therapy of the Prostate," Physics in Medicine and Biology, Vol. 56, No. 16, 2011, pp. 52875302. doi:10.1088/0031-9155/56/16/014

[6] Y. K. Lim, J. Kwak, D. W. Kim, D. Shin, M. Yoon, S. Park, J. S. Kim, S. H. Ahn, J. Shin and S. B. Lee, "Microscopic Gold Particle-Based Fiducial Markers for Proton Therapy of Prostate Cancer," International Journal of 
Radiation Oncology, Biology, Physics, Vol. 74, No. 5, 2009, pp. 1609-1616. doi:10.1016/j.ijrobp.2009.02.076

[7] R. J. Kudchadker and A. K. Lee, "Effectiveness of Using Fewer Implanted Fiducial Markers for Prostate Target Alignment," International Journal of Radiation Oncology, Biology, Physics, Vol. 74, No. 4, 2009, pp. 1283-1289. doi:10.1016/j.ijrobp.2009.02.033

[8] A. Lomax, "Intensity Modulation Methods for Proton Radiotherapy," Physics in Medicine and Biology, Vol. 44, No. 1, 1999, pp. 185-205. doi:10.1088/0031-9155/44/1/014

[9] J. O. Deasy, D. Shepard and T. R. Mackie, "Distal Edge Tracking: A Proposed Delivery Method for Conformal Proton Therapy Using Intensity Modulation," Proceedings XIIth ICCR, Salt Lake City, 1997.

[10] M. Zhang, D. Westerly and T. Mackie, "Introducing an On-Line Adaptive Procedure for Prostate Image Guided Intensity Modulate Proton Therapy," Physics in Medicine and Biology, Vol. 56, No. 15, 2011, pp. 4947-4965. doi:10.1088/0031-9155/56/15/019
[11] A. Niemierko, "Reporting and Analyzing Dose Distributions: A Concept of Equivalent Uniform Dose," Medical Physics, Vol. 24, 1997, pp. 103-110. doi:10.1118/1.598063

[12] Y. Liao, M. Joiner, Y. Huang and J. Burmeister, "Hypofractionation: What Does It Mean for Prostate Cancer Treatment?" International Journal of Radiation Oncology, Biology, Physics, Vol. 76, 2010, pp. 260-268. doi:10.1016/j.ijrobp.2009.06.043

[13] J. Lyman and A. Wolbarst, "Optimization of Radiation Therapy, IV: A Dose-Volume Histogram Reduction Algorithm," International Journal of Radiation Oncology, Biology, Physics, Vol. 17, No. 2, 1989, pp. 433-436. doi:10.1016/0360-3016(89)90462-8

[14] W. Tome and J. Fowler, "On Cold Spots in Tumor Subvolumes," Medical Physics, Vol. 29, No. 7, 2002, pp. 1590-1598. doi:10.1118/1.1485060

[15] http://physics.nist.gov/cgi-bin/Star/compos.pl?ap 